Questions vives

\section{Questions Vives}

Recherches en éducation

$n^{\circ} 22 \mid 2014$

Questionner le curriculum en éducation physique et sportive : Quelle dynamique en contexte? Quelle autonomie?

\title{
Curriculum en Education Physique et Sportive et évaluation certificative au baccalauréat
}

Curriculum in Physical Education and Sports and certification assessment in

baccalaureate

Stéphane Brau-Antony et Benoît Hubert

\section{(2) OpenEdition}

1 Journals

Édition électronique

URL : http://journals.openedition.org/questionsvives/1596

DOI : 10.4000 /questionsvives. 1596

ISSN : 1775-433X

Éditeur

Université Aix-Marseille (AMU)

Édition imprimée

Date de publication : 15 décembre 2014

ISBN : 978-2-912643-46-9

ISSN : $1635-4079$

Référence électronique

Stéphane Brau-Antony et Benoît Hubert, « Curriculum en Education Physique et Sportive et évaluation certificative au baccalauréat », Questions Vives [En ligne], n²2 | 2014, mis en ligne le 16 février 2015, consulté le 19 avril 2019. URL : http://journals.openedition.org/questionsvives/1596; DOI : 10.4000/ questionsvives. 1596

Ce document a été généré automatiquement le 19 avril 2019.

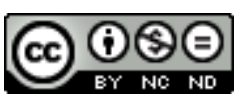

Questions Vives est mis à disposition selon les termes de la licence Creative Commons Attribution -

Pas d'Utilisation Commerciale - Pas de Modification 4.0 International. 


\section{Curriculum en Education Physique et Sportive et évaluation certificative au baccalauréat}

Curriculum in Physical Education and Sports and certification assessment in baccalaureate

Stéphane Brau-Antony et Benoît Hubert

\section{Introduction}

Cet article se propose d'étudier le curriculum en actes en Education Physique et Sportive (EPS) en l'examinant du point de vue des pratiques d'évaluation des enseignants et plus particulièrement dans le cadre de la certification au baccalauréat. Ce mode d'entrée dans l'analyse du curriculum peut paraître inhabituel dans la mesure où le curriculum en actes se donne à voir principalement à travers ce qui s'enseigne et ce qui s'apprend au fil des séances. Ce point de vue est notamment développé par Lenoir (2006) mais aussi par Dolz, Jacquin et Schneuwly (2006) qui montrent l'intérêt d'analyser comment le curriculum prescrit se traduit sur le terrain scolaire : quel est le curriculum effectivement enseigné et quelles sont les conditions de son implémentation dans les situations quotidiennes de classe ? Pour autant, un certain nombre de travaux soulignent que porter un regard sur le curriculum tel qu'il s'évalue peut aussi être envisagé (voir à ce sujet le numéro 25 de la revue Les Dossiers des Sciences de l'Education intitulé Evaluation et curriculum, 2011). C'est pourquoi Lenoir (2011) estime que l'évaluation peut être considérée dans une double perspective, celle des apprentissages réalisés par les élèves et celle de la mise en œuvre du curriculum prescrit et des décisions relatives à son évaluation. Il est ainsi possible de s'interroger sur le curriculum acquis par les élèves (quelles sont les traces des curricula prescrits et enseignés chez les élèves ?) mais aussi sur les pratiques d'évaluation du curriculum telles que le met en évidence Grisay (1989) concernant l'élaboration des épreuves d'examen. L'entrée par la certification pour traiter du curriculum évalué 
renvoie ainsi à la nature des épreuves choisies, aux critères et indicateurs d'évaluation retenus, à l'échelle de notes ou d'appréciation, aux éléments du curriculum faisant l'objet d'une évaluation (De Ketele, 2011). C'est dans cette dernière perspective que nous situerons notre contribution en nous intéressant à la certification en EPS pour les classes de lycées général et technologique.

2 Depuis 2002 la certification au baccalauréat est pilotée par des référentiels d'évaluation nationaux dans différentes Activités Physiques Sportives et Artistiques (APSA). Les référentiels sont explicitement organisés à partir du curriculum formel, ils identifient des degrés d'acquisition du niveau de compétence attendue (Bulletin Officiel du 19 juillet 2012) et à ce titre ils permettent de préciser les productions motrices visées de fin d'enseignement secondaire (ce que devrait être un élève physiquement éduqué), les compétences constituant les objets à évaluer. En proposant des référentiels nationaux le législateur a ainsi voulu éviter un certain nombre d'écueils soulevés par les textes précédant ceux de 2002. L'absence d'équité entre les candidats liée au fait que les équipes pédagogiques avaient l'initiative de construire leur propre référentiel ainsi que la faible objectivité de l'évaluation, sauf pour les APSA disposant d'un barème, conduisaient les enseignants à se livrer à une série d'arrangements évaluatifs (David, 2000) peu propices à une évaluation rigoureuse du curriculum prescrit. Les prescriptions en matière de certification sont désormais très fortes. Sont spécifiés l'épreuve d'évaluation, les critères et indicateurs de l'évaluation relatifs aux niveaux de compétences visés, les repères sur l'échelle de mesure ( $\mathrm{cf}$ annexe). Par ailleurs l'évaluation se déroule en doublette puisque deux enseignants dont le responsable de la classe évaluent les prestations des élèves. L'activité certificative de l'enseignant est donc soumise à un système de contraintes qui laisserait penser qu'il dispose de peu de marge de manœuvre pour évaluer la maitrise par les élèves du contenu du curriculum. Pour autant, nous avancerons l'hypothèse selon laquelle les enseignants d'EPS se dotent d'une certaine autonomie vis-à-vis des prescriptions auxquelles ils doivent s'astreindre : quelles distances prennent-ils avec les injonctions qui leur sont faites? Comment se manifestent ces écarts avec les prescriptions officielles ? Le curriculum évalué correspond-il au curriculum prescrit ?

3 Après un rappel des travaux de recherches portant sur la certification en EPS en lien avec le curriculum, nous caractériserons l'activité évaluative des enseignants en convoquant les cadres et les outils de l'analyse du travail (ergonomie et clinique de l'activité). Nous souhaitons décrire et comprendre cette activité évaluative et notamment les décisions prises par les enseignants lorsqu'ils utilisent les référentiels de certification. Il s'agit d'explorer l'activité réelle des évaluateurs notamment quand ils sont confrontés à l'évaluation du curriculum prescrit tel qu'il apparaît dans les référentiels. Nous nous intéressons à la manière dont les enseignants interprètent les prescriptions qui leur sont fournies, à ce qu'ils en font en fonction des moyens dont ils disposent, des exigences qu'ils se donnent face aux contraintes auxquelles ils ont à faire face.

4 Afin de documenter l'activité des évaluateurs et rendre intelligible le curriculum tel qu'il s'évalue, nous rendrons compte d'une recherche ayant utilisé la technique de l'instruction au sosie auprès de huit enseignants de lycée qui évaluent en sports collectifs. 


\section{Le cadre conceptuel}

\subsection{Les recherches sur la certification en EPS}

5 Les recherches sur la certification en EPS n'abordent pas de front la question du curriculum ou alors de façon détournée. En s'intéressant à l'élaboration des barèmes Cleuziou (1996) interroge leur étalonnage. Selon l'auteur évaluer les prestations motrices des élèves c'est construire un système de conventions qui puisse déterminer les performances attendues en fonction d'une population de référence. Si l'exercice peut paraître aisé dans des épreuves mesurées (athlétisme ou natation), il s'avère beaucoup plus délicat dans des APSA comme les sports collectifs notamment quant il s'agit de cerner les contours des niveaux de compétences attendus en collège ou en lycée et de leur faire correspondre une note. Ce balisage des niveaux d'exigence est d'autant plus difficile à élaborer que les normes d'excellence (Perrenoud 1984) qui représentent l'image d'une pratique idéale parfaitement maîtrisée ne découlent pas directement des programmes, elles n'y sont d'ailleurs nullement consignées par écrit ou alors elles y figurent de manière implicite.

Les travaux de David (2000) tentent de jeter les bases d'une théorie de l'évaluation certificative en l'abordant du point de vue des représentations des enseignants et des procédures d'évaluation mobilisées. L'auteur montre que les enseignants de lycée doutent que les objectifs qui sont assignés à l'évaluation aux examens puissent permettre de faire un inventaire des acquis des élèves au cours de leur scolarité. La certification constitue selon eux une "approximation risquée » (Cardinet, 1987) au point qu'ils remettent en cause l'idée que cette évaluation rend compte de ce qui a été enseigné. Ce constat est corroboré par Brau-Antony (2000) qui met en évidence les obstacles rencontrés par les enseignants pour définir les produits attendus et les normes d'excellence à partir de l'exemple des sports collectifs. Les normes d'excellence sont bien souvent calquées sur le modèle de la haute performance. Les produits évalués sont révélateurs d'un modèle d'excellence proche de l'activité mobilisée par un joueur de haut niveau en décalage avec ce que l'on peut attendre des élèves en contexte scolaire. L'excellence motrice est définie en fonction de ce que réalise l'expert et non pas en fonction de ce que pourrait accomplir un élève de Terminale compte tenu des contraintes du temps didactique. Tout se passe donc comme si la définition des normes d'excellence scolaire mettait les enseignants d'EPS en difficulté si bien que l'excellence évaluée est le plus souvent externe à l'école. La définition des performances scolaires soulève ainsi de nombreuses interrogations car il est nécessaire de situer les performances des élèves sur un continuum évaluatif en étant capable d'identifier leurs prestations aux extrémités de ce continuum (prestation de faible niveau versus prestation de haut niveau) sachant que de nombreux apprentissages se déroulent en dehors de l'école et que les meilleurs élèves en EPS ont souvent appris au sein des clubs ou ailleurs dans les pratiques non institutionnalisées. Il existe ainsi un autre curriculum de formation qui interfère avec le curriculum scolaire, qui vient brouiller ce que les élèves ont réellement appris dans leurs cursus scolaire et par conséquent ce qu'il convient d'évaluer. 


\subsection{L'activité enseignante : du travail prescrit au travail réel}

7 Les recherches en ergonomie du travail enseignant "consistent à affirmer que les principes de l'action des enseignants ne résident pas uniquement dans la situation où se déroule l'interaction, mais qu'il faut les rechercher aussi dans les prescriptions et dans l'activité d'interprétation et de redéfinition à laquelle elles donnent lieu de la part des enseignants » (Saujat, 2002 , p. 63). L'écart entre ce qui est demandé aux enseignants et ce qu'ils font réellement est au fondement même de l'approche ergonomique (Amigues \& Lataillade, 2007).

Identifier ainsi les déterminants de l'activité enseignante c'est les rapporter aux sources de prescriptions qui vont affecter les prises de décision de l'enseignant. On peut ainsi repérer différents niveaux de prescriptions :

- celles qui relèvent des prescriptions descendantes (Daniellou, 2002); ce sont des prescriptions exogènes constituées par les recommandations issues des programmes mais aussi les référentiels de certification. Ces prescriptions renvoient à la dimension impersonnelle du métier (Clot, 2013) qui précise, codifie et organise en surplomb ce qu'il y a à faire. Les prescriptions officielles s'accompagnent parfois d'autres types de prescriptions qui proviennent des milieux de la formation ;

- celles qui font l'objet d'une réorganisation opérée par les professionnels. Les prescriptions descendantes subissent un certain nombre de transformations qui font office de règles pour le collectif de professionnels. Elles sont qualifiées de prescriptions remontantes (Six, 1999) ;

- celles qui relèvent de la personnalisation de ces prescriptions par chaque enseignant qui s'auto-prescrit ce qu'il y a à faire.

Etudier l'activité évaluative des enseignants c'est donc se pencher sur la dynamique qui articule ces trois niveaux de prescriptions et notamment tenter de décrire et comprendre les arbitrages auxquels se livrent les enseignants. Les enseignants procèdent ainsi à des compromis entre ce qu'on leur demande de faire et les exigences des tâches auxquelles ils ont à faire face lorsqu'ils évaluent. Ceci nous conduit à investiguer au plus près l'activité réelle de l'évaluateur en convoquant les travaux en clinique de l'activité qui, dans la continuité de ceux de l'ergonomie, montrent que l'activité d'un professionnel est beaucoup plus riche que les injonctions et préconisations qui lui sont faites. L'activité du sujet même si elle en porte la marque ne peut se réduire à des prescriptions descendantes, en ce sens il y a toujours des écarts entre le travail prescrit et le travail réalisé (Leplat, 1997). Pour autant, si l'étude du travail réalisé par l'enseignant nous semble éminemment utile, elle ne permet de dévoiler qu'une partie de l'activité, celle-ci étant le résultat d'une lutte entre plusieurs actions possibles (Vygotski, 1978). L'activité réalisée n'est pas uniquement regardée comme le résultat de l'intention d'un sujet qui agirait de façon rationnelle, ce qui occulterait la part subjective de son activité. C'est une activité où le sujet doit faire des choix qui entrent en concurrence, une sorte de conflit réel que l'activité réalisée ne résout jamais complètement. L'activité réelle c'est ce que l'on fait, mais également ce qu'on ne fait pas et que l'on aurait voulu ou pu faire, l'activité devient alors empêchée, contrariée, avortée, parsemée de renoncements (Clot, 2008).

C'est dans cette perspective que la clinique de l'activité se donne pour but d'élargir le pouvoir d'action des sujets sur leur propre activité. Il s'agit d'une part de comprendre le travail pour le transformer et d'autre part de le transformer pour en comprendre le développement. Cet objectif implique la mise en place de cadres dialogiques (méthodes 
d'autoconfrontation croisée) afin de favoriser la prise de parole des sujets sur leur propre travail. Clot (2008) ne considère pas cette méthode uniquement comme un instrument d'explicitation et de formalisation de l'expérience mais comme un instrument de développement de l'expérience. Le but est ainsi d'organiser les conditions d'un redoublement de l'expérience vécue: le dialogue avec un autre professionnel est l'occasion de dialoguer avec soi même. Dans ce cas, les méthodes utilisées cherchent à solliciter chez les professionnels une activité d'observation et d'interprétation de leur situation de travail de manière à faire surgir des désaccords, des controverses sur la qualité du travail réalisé mais aussi des dilemmes, des tensions, des conflits rencontrés dans l'exercice du métier.

11 Se pose ici la question de l'usage des méthodes d'autoconfrontation qui sont habituellement mises en place dans des dispositifs de recherche-intervention à la demande de collectifs de travail à des fins de développement de l'activité. L'hypothèse sous-jacente est que les transformations du travail ne peuvent être portées que par les professionnels eux-mêmes. Selon Clot (2008) le chercheur en clinique de l'activité peut cependant mener deux projets en poursuivant des objectifs distincts : si un professionnel cherche à optimiser sa pratique grâce à une réflexion, le chercheur en clinique de l'activité peut l'aider et lui permettre de s'expliquer sur son activité. Le premier pourra éventuellement se servir de cette objectivation ou prise de recul sur son action pour faire autrement s'il le souhaite ou s'il le peut. Le second dans son activité de recherche, visera à comprendre comment ce professionnel développe son expérience. Le chercheur a donc ici pour objectif de produire des connaissances sur une activité professionnelle particulière. C'est dans cette seconde perspective que nous nous situons. Considérant ainsi que l'évaluation des prestations des élèves est une composante importante de la professionnalité des enseignants d'EPS de lycée (Carnus \& Terrisse, 2006) nous cherchons à documenter leur activité évaluative, à en saisir l'épaisseur, la complexité ainsi que sa dimension subjective.

\section{Méthodologie}

12 Afin d'accéder à l'activité réelle des enseignants et de rendre visible leur activité évaluative nous avons utilisé l'instruction au sosie (IS). Ce type d'entretien repose sur la co-analyse d'une situation de travail entre deux personnes: l'instructeur et le sosie (Oddone, Rey \& Briante, 1981). L'objectif est de conduire l'instructeur qui s'adresse à son remplaçant potentiel, le sosie, à être confronté à son expérience en dialoguant avec un autre. Il s'agit d'inciter l'instructeur à regarder son expérience avec les yeux du sosie dans la mesure où le sosie ne peut pas la voir de la même façon que l'instructeur. Répondre aux questions du sosie oblige ainsi l'instructeur à rendre compte de son travail et à l'amener suite aux questions du sosie, à exprimer des éléments qu'il n'aurait pas pensé verbaliser.

Quatre IS ont été réalisées, huit enseignants de lycée ont été sollicités (six femmes et deux hommes âgés de 38 à 57 ans). L'ensemble de ces enseignants présente une stabilité de poste dans l'affectation en lycée de plus de dix ans. L'IS mettait en présence deux enseignants exerçant dans des établissements différents afin d'éviter toute connivence. La consigne délivrée par le chercheur était la suivante : « suppose que je sois ton sosie et que demain je me trouve en situation de te remplacer dans ton travail. Quelles sont les instructions que tu devrais me transmettre afin que personne ne s'avise de la substitution?». L'instructeur devait utiliser le «tu» et le sosie le «je». La situation de 
travail était spécifiquement centrée sur la séance d'évaluation terminale au moment où les deux enseignants évaluent les productions des élèves à l'aide du référentiel de certification. Deux IS ont porté sur l'évaluation en volley-ball, les deux autres concernaient le handball.

Les quatre IS ont été transcrites verbatim. Le traitement des données a consisté à séquencer les IS, une séquence étant constituée d'un certain nombre de tours de parole instructeur/sosie relatifs à un objet de discours particulier (Hubert, 2013) : les segments d'IS choisis dans la présentation des résultats font état de la motricité du dialogue entre les deux enseignants afin de rendre compte de la dynamique des interactions entre les deux interlocuteurs. Nous n'avons retenu que les objets de discours (ce dont il est question dans les IS) qui sont en lien direct avec l'évaluation du curriculum, par exemple les aspects organisationnels de l'évaluation relatifs à la constitution des équipes, à la gestion des effectifs des classes ainsi qu'à la prise en compte des contraintes matérielles ont été laissés de côté. Par ailleurs, nous avons également pointé les moments où un objet de discours peut se trouver reconverti en objet de débat notamment quand les deux enseignants font état de désaccords suscitant une controverse professionnelle.

\section{Résultats}

Les résultats font apparaître trois préoccupations majeures qui sont systématiquement présentes dans les quatre IS :

- la modification des exigences prescrites par les référentiels de certification;

- la possibilité ou pas d'évaluer d'autres types de compétences que celles qui sont inscrites dans le curriculum ;

- le problème de l'évaluation des niveaux de jeu des filles et des garçons lié à l'indifférenciation des critères et indicateurs.

Ces préoccupations concernent le choix des objets d'évaluation et reflètent les débats engagés entre les professionnels autour des marges de liberté qu'ils se donnent dès qu'il s'agit de prendre des distances avec ce que prescrit le référentiel.

\subsection{Une révision des exigences par rapport à certains profils d'élèves}

17 «Elèves frontières » (expression utilisée dans l'IS $n^{\circ} 4$ ), élèves en difficulté (IS $n^{\circ} 2:$ « dans une activité qui est prise par défaut (...) «s'ils ne savent pas nager, il sont obligés de prendre volley »), élèves de très bon niveau, élèves perturbateurs, élèves ayant été absents une partie du cycle d'apprentissage ... sont autant de profils d'élèves qui alimentent les échanges entre les deux professionnels et provoquent parfois des débats au sein des IS dans la mesure où les référentiels d'évaluation ne tiennent pas compte des caractéristiques spécifiques des élèves. Ce sont pourtant des situations récurrentes auxquelles sont confrontés les enseignants.

18 L'extrait suivant (IS $n^{\circ} 4$ ) aborde le problème du niveau des élèves en rapport avec les exigences visées par les programmes et les compétences qui s'y rapportent : le référentiel institutionnel fixe un niveau de compétence attendu (le niveau 4) et spécifie grâce à des 
critères et indicateurs le niveau 4 non acquis (en dessous de 10) et deux degrés d'acquisition du niveau 4 (de 10 à 16 et de 17 à 20). Les éléments à évaluer ont trait à :

- la pertinence et l'efficacité de l'organisation collective pour huit points ;

- l'efficacité collective (gain des rencontres) pour deux points ;

- l'efficacité individuelle du joueur dans l'organisation collective pour huit points.

Instructeur (I) : « ... sur certaines ... parce qu'il y a des choses qui ... tu verras, enfin comment dire qui sont prioritaires dans ce qui est appris aux élèves qui semblent voilà quoi plus prioritaires que d'autres.

Sosie (S) : alors est-ce que je dois interpréter que mon cycle doit être construit non pas forcément par rapport au cadre d'évaluation mais par rapport aux attentes.

I : non le cadre est respecté mais les choses sont travaillées en fonction des attentes.

$\mathrm{S}:$ mais est-ce que je dois réviser en fonction des attentes ? [...].

I : c'est une décision que je m'applique, que tu feras. Il y a le cas par exemple des élèves frontières ... j'y tiens!... enfin ... t'y tiendras! Donc, t'auras des élèves qui sont pas mal donc ... tu feras ... enfin, tu verras quand tu auras la liste des élèves tu feras comme tout le monde ... un moment donné quand il t'en faut 6 ou il t'en faut 7 et il t'en faut pas 18 tu vois pour faire euh ... tu triches sur les ... euh tu t'organiseras, tu verras ... ça sera organisé aussi par rapport au nombre d'élèves. Des élèves qui sont pas mal et qui sont dans le groupe plus fort mais qui sont «justos » quoi tu vois? Et qui ont besoin d'un bonus parce qu'ils sont pas mal mais ils sont pas forcément pile poil de ce niveau là mais il t'en faut 18 là ... eh bien voilà ! Donc, tu leur fais faire, en dehors, des tournois complètement en dehors des tournois de certification pour les deux points là ... d'accord? Tu fais un match ou deux de bonus où les élèves frontières vont pouvoir faire monter leur curseur ou pas en nous montrant dans le duel, dans un rapport au gardien, dans des contre-attaques où les élèves reviennent moins vite etc. euh ... on va dire des compétences ».

Les interactions entre les deux enseignants portent sur l'écart entre les compétences visées par le curriculum qui relèvent des prescriptions exogènes et les prescriptions endogènes, celles que doit se fixer l'enseignant quand il doit certifier les niveaux de compétence de «ses» élèves en handball. L'instruction donnée au sosie porte sur les attentes c'est-à-dire sur ce qu'il faut évaluer en termes de priorités dans « ce qui est appris aux élèves». L'interrogation du sosie fait état d'un trouble voire d'une résistance qui renvoie à un véritable dilemme : est-il possible de s'affranchir du "cadre d'évaluation" alors que le cycle d'apprentissage doit être organisé en fonction des possibilités des élèves sachant que les objectifs recherchés par l'enseignant (" en fonction des attentes » selon les propos de l'instructeur) peuvent être en décalage avec les niveaux d'exigence prévus par le référentiel ? Peut-on rendre compatibles ses propres attentes, compte-tenu du niveau réel des élèves, avec les injonctions du législateur? Le cas des "élèves frontières » abordé par l'instructeur indique comment on peut envisager de se sortir de ce dilemme en "trichant ». En effet certains élèves n'entrent pas facilement dans les caractérisations des niveaux de jeu définies par le référentiel. Ces "élèves frontières » se situent dans un entredeux qui ne leur permet pas d'accéder au groupe de niveau supérieur car ils sont considérés comme «justos» et ne sont pas "forcément pile poil de ce niveau là». Cette situation pousse l'enseignant à proposer un tournoi parallèle au tournoi officiel tel qu'il est prévu par les textes pour affiner son évaluation et permettre à des élèves d'être valorisés pour passer au niveau supérieur. Il s'agit de faire bouger «le curseur ", de créer les conditions pour que ces élèves puissent franchir un palier même si on déroge aux principes d'élaboration de l'épreuve d'évaluation: l'équité entre les élèves suppose que chaque équipe dispute le même nombre de rencontres et donc que certains élèves ne 
bénéficient pas d'un traitement de faveur. Les compétences évaluées le sont donc dans un environnement plus facilitateur ( dans des contre-attaques où les élèves reviennent moins vite») où le rapport de forces leur est plus favorable grâce à un détournement des prescriptions officielles. C'est pour cet enseignant le prix à payer pour permettre que les compétences évaluées soient en accord avec ses propres objectifs tout en ne contestant pas radicalement de ce qui figure dans les référentiels.

Ce dialogue entre les deux enseignants interroge le modèle de référence (Noizet \& Caverni, 1978) de l'évaluateur et plus particulièrement la définition du produit attendu et du produit norme dans des APSA comme les sports collectifs. Au motif que le temps scolaire consacré aux apprentissages est insuffisant, que les conditions d'enseignement sont souvent difficiles et que le niveau des élèves est très hétérogène, les enseignants d'EPS estiment que les compétences attendues par les programmes sont peu réalistes (David \& Brau-Antony, 2002) si bien qu'ils ne s'autorisent pas à sanctionner les élèves en mettant de mauvaises notes. Dans les quatre IS les enseignants s'accordent pour que les notes ne soient pas trop basses, les notes se concentrant entre 07/08 et 20, même si le niveau 4 non acquis correspond à une note entre 0 et 09 . Il apparaît ainsi que c'est avant tout la note qui pilote l'évaluation; les enseignants ne s'assurent pas toujours que les élèves maîtrisent les compétences du référentiel en prenant en compte la totalité des éléments à évaluer dans l'efficacité collective et l'efficacité individuelle. L'essentiel est ailleurs comme le souligne l'extrait de l'IS $n^{\circ} 3$, il s'agit de présenter des notes acceptables au regard des normes en usage dans la discipline EPS tout en sachant qu'une commission d'harmonisation statue en dernier ressort sur les notes délivrées par les équipes d'établissement.

Extrait IS $\mathrm{n}^{\circ} 3$ :

$\mathrm{I}:$ « tu n'hésite pas à mettre 20.

$\mathrm{S}$ : donc je vais avoir ... donc je monte à 20 .

I : ça ne doit pas te déranger de mettre 20.

$\mathrm{S}:$ donc entre 08 et 20 avec une moyenne qui tourne autour de 13 ?

I : oui 13-14 c'est souvent ça.

$\mathrm{S}$ : donc mes notes sont balisées comme ça.

I : oui euh sur l'amplitude ... ».

3.2. Des objets d'évaluation en dehors du curriculum prescrit

Dans l'ensemble des IS un des objets de discours porte sur la prise en compte d'autres éléments à évaluer que ceux figurant dans les référentiels. Ces éléments relèvent d'une part des comportements manifestés par les élèves au cours du cycle (IS n ${ }^{\circ} 2$ ), d'autre part ils concernent les dimensions techniques spécifiques aux sports collectifs que les référentiels ont tendance à occulter (IS $\mathrm{n}^{\circ} 1$ ).

\subsubsection{Tenir compte de l'investissement des élèves}

Extrait IS $n^{\circ} 2$ :

$\mathrm{S}:$ : et l'élève qui a bien travaillé, qui a progressé?

I : tu te rends compte que là il a pas été à son niveau.

$\mathrm{S}$ : ça veut dire que je dois tenir compte de l'investissement de l'élève, de son travail pendant le cycle?

I : oui en quelque sorte, ça arrive d'avoir ... d'avoir ... l'idée de dire, tiens c'est vache quand même, il a ... il a bien bossé et puis aujourd'hui il « ramasse ». 
$\mathrm{S}$ : oui il y a le professeur qui a vu tous les progrès et puis il y a l'œil qui est extérieur et c'est une moyenne, une négociation entre les deux. Donc il y a le facteur humain qui est important et pourquoi pas en EPS, même si c'est une évaluation de bac ... on est quand même sur du contrôle en cours de formation et non pas sur du contrôle ponctuel ».

Cet extrait montre les tensions auxquelles sont soumis les enseignants lors de l'évaluation terminale. Les enseignants sont tenus dans une optique certificative de se prononcer le jour de l'épreuve d'évaluation sur le niveau atteint par les élèves quelle que soit la qualité de leur travail fourni au cours du cycle. La question posée par le sosie : " et l'élève qui a bien travaillé, qui a progressé?"», soulève une interrogation concernant d'autres éléments qui pourraient être évalués en EPS. Il est ainsi question de "tenir compte de l'investissement de l'élève, de son travail pendant le cycle » alors que les référentiels de certification ne font pourtant pas mention de postures ou d'attitudes face au travail que les enseignants considèrent comme déterminantes pour les apprentissages. Tout se passe comme si à côté de ce curriculum prescrit et évalué qui met principalement l'accent sur les compétences techniques et tactiques des sports collectifs existait un autre type de curriculum qui renverrait à d'autres objets d'enseignement qui, selon les enseignants, mériteraient d'être évalués. De fait l'enseignant ne peut ignorer l'investissement et la participation des élèves, que le contrôle en cours de formation devrait pouvoir valoriser (« on est quand même sur du contrôle en cours de formation et non pas sur du contrôle ponctuel »).

Une partie du curriculum évalué ne correspond donc pas aux prescriptions institutionnelles, les enseignants n'hésitent pas à procéder à un certain nombre d'arrangements (Merle, 1996), au point que la note ne reflète pas toujours les prestations des élèves réalisées lors du contrôle terminal: on note aussi autre chose, par exemple «l'élève qui a bien bossé » et l'on s'appuie pour ce faire sur « le professeur qui a vu tous les progrès » car il a eu la responsabilité de la classe sur tout le cycle. Ainsi lorsque l'institution ne prévoit pas de prendre en compte certaines dimensions de l'activité de l'élève comme c'est le cas aujourd'hui avec la disparition de la note de participation (présente sous des formes diverses jusqu'en 2002), on constate que les enseignants contournent les dispositions officielles et réintroduisent des dimensions qui conditionnent les progrès individuels et collectifs. La note fait office de variable d'ajustement de façon à faire correspondre le curriculum évalué avec les priorités que se donnent les enseignants. On augmente la note de l'élève qui a fait des efforts en le "boostant un petit peu " (IS n ${ }^{\circ}$ ) ou alors «si le gamin il a travaillé tout le temps ... tu n'es pas là pour les enfoncer, tu n'es pas là pour mettre une note qui n'est pas révélatrice du travail qu'il a fait tout au long de l'année " ( $\mathrm{I}$ : IS $n^{\circ} 2$ ). On n'hésite pas à l'inverse à sanctionner l'élève qui ne s'est pas impliqué pendant les cours.

\subsubsection{Evaluer des éléments techniques non prévus par le référentiel}

Le curriculum tel qu'il s'évalue subit parfois des transformations notables notamment quand les enseignants interprètent le contenu des référentiels. En effet les concepteurs des référentiels "modélisent " les objets évalués à partir de leur propre système de références (Figari, 1994). Les référentiels traduisent ainsi une certaine conception des sports collectifs (Brau-Antony, 2001) qui oriente le choix des objets à évaluer. Ces choix peuvent être contestés par les enseignants si bien que l'évaluation des niveaux de jeu des élèves peut porter sur des objets qui se distinguent de ceux qui sont sélectionnés dans les prescriptions officielles. 
$\mathrm{S}$ : «est-ce que je dois évaluer par rapport à son engagement, sa technique parce que avec mon collègue Bertrand on insiste beaucoup sur le service et le smash (...) s'il smashe plein de fois il a 20.

I : c'est pas une grille où on met plein de croix quand telle action est réalisée, est faite et ... réalisée ou pas.

$\mathrm{S}$ : il peut avoir raté son smash.

I : il peut avoir raté son smash, c'est-à-dire à un moment donné, est-ce qu'il était en position de smasher ou pas, est-ce qu'il a tenté un geste pas très cohérent par rapport à sa place sur le terrain, par rapport à comment il est placé par rapport au ballon, si je me retrouve à smasher et que je suis complètement tendu vers l'arrière, voilà ça a pas forcément d'intérêt. Ya aussi est-ce qu'il a smashé en sautant sur le dos de son camarade ou au contraire est-ce qu'il était vraiment en position de smasher. Il y a tout ça à prendre en compte, quoi, est-ce qu'il prend en considération ses camarades ou est-ce qu'il est le seul sur le terrain, il y a que lui qui joue ».

Cet échange fait état d'un désaccord entre les deux protagonistes et prend la forme d'une controverse autour des objets d'évaluation qu'il faut privilégier. Le sosie met en exergue l'importance du service mais surtout du smash, ce point de vue est renforcé par celui de son collègue Bertrand qui est mentionné dans l'IS. Le smash est ici entendu comme une composante technique essentielle à laquelle on ne peut se soustraire quand on évalue en volley-ball puisqu'il fait partie du répertoire gestuel du volleyeur ("avec mon collègue Bertrand on insiste beaucoup sur le service et le smash»). Il convient ainsi de vérifier la réussite au smash de même que sa fréquence d'apparition dans le jeu ( $~ i l$ peut avoir raté son smash "; " s'il smashe plein de fois il a 20 »). Signalons que le terme smash n'apparait pas dans le référentiel de certification, il est question de choix pertinent pour l'attaquant afin de rompre l'échange en jouant sur les paramètres liés à la direction de la frappe et à sa vitesse, à ce titre le smash peut constituer un choix tactique adapté en fonction de la configuration de la situation. C'est dans cette perspective que se situe l'instructeur. Le smash n'est pas selon lui un objet d'évaluation en tant que tel, une forme gestuelle repérable et quantifiable: "c'est pas une grille où on met plein de croix quand telle action est réalisée ». Ses propos témoignent d'une résistance vis-à-vis d'une conception du jeu développée par le sosie qui ne place pas le smash comme une solution possible à un problème posé par le jeu. Selon l'instructeur cela ne peut pas être une forme gestuelle qui prendrait le statut d'objet d'enseignement versus objet d'évaluation. Le choix de smasher dépend d'une part du placement du joueur sur le terrain (situation favorable ou pas), de la nécessaire coordination de l'action du joueur avec celles de ses partenaires ( est-ce qu'il prend en considération ses camarades ou est-ce qu'il est le seul sur le terrain?»), enfin de la réorganisation posturale que nécessite la décision de smasher («par rapport à comment il est placé par rapport au ballon »). En ce sens il se met en opposition avec le sosie sans pour autant le convaincre.

Si la fonction du référentiel est d'afficher ce que le législateur attend d'un élève de Terminale en volley-ball, le débat entre les deux enseignants indique que le choix des objets d'évaluation revêt un enjeu crucial pour eux car il traduit leurs conceptions des savoirs à enseigner et à évaluer dont dépend la construction du curriculum. Cela montre que lorsque l'enseignant prend un certain nombre de décisions, il recourt à une théorie implicite mêlant des conceptions, croyances, représentations, connaissances technologiques, savoirs professionnels divers qui orientent sa pratique professionnelle. Sensevy (2007) parle d'épistémologie pratique afin de souligner que cette théorie d'action à usage professionnel peut avoir des conséquences pratiques sur le pilotage de la classe et 
plus particulièrement sur le système didactique lui-même. On peut faire l'hypothèse que la controverse mise en évidence dans l'IS n 1 relève aussi de l'épistémologie pratique des deux évaluateurs quand ils débattent de ce qu'il est important d'évaluer en volley-ball.

\subsection{Evaluer le niveau de jeu des filles en le comparant avec celui des garçons}

31 L'extrait suivant (IS ${ }^{\circ}{ }^{\circ}$ ) aborde la question des notes des filles et des garçons dans l'APSA handball où le barème n'existe pas contrairement à une APSA comme l'athlétisme qui distingue les performances des filles et des garçons.

$\mathrm{S}:$ « avec aucune différence garçons/filles?

I: Si. Tu fais une différence garçons/filles, tu n'hésites pas par rapport à un même niveau de prestation.

$\mathrm{S}:$ motricité de fille, motricité de garçon.

I : tu n'hésites pas à...

$\mathrm{S}$ : et à quel moment est-ce que je vais plutôt le nuancer, ça va être plutôt dans l'efficacité individuelle?

I : oui sur l'aspect individuel, tu n'hésites pas à monter ...1 ou 2 points sur la note.

$\mathrm{S}$ : ce qui fait qu'à la fin il y a un petit écart mais qui est moindre. L'écart garçons/filles, moyenne des garçons, moyenne des filles.

I : il y un petit écart mais ...

$\mathrm{S}$ : qui est moindre.

I : qui n'est pas important.

$\mathrm{S}$ : avec la même bonne volonté, on devrait arriver à des notes de même niveau normalement s'il y avait le même investissement.

I : s'il y a le même investissement, même si au niveau de la motricité c'est un petit moins ... on arrive à la même chose.

$\mathrm{S}$ : oui sur la puissance des tirs.

I : exactement.

$\mathrm{S}:$ la puissance des passes.

I : c'est important ce que tu dis sur les filles parce que effectivement, des fois on a tendance à l'oublier un petit peu.

$\mathrm{S}: \grave{a}$ avoir cette vision du handballeur.

I : même en sports co parce que tu regardes, ça n'apparaît nulle part.

$S:$ non parce que c'est pas facile à écrire.

I : c'est pas simple en même temps.

$\mathrm{S}$ : c'est pas simple à écrire.

I : faut que ça soit pris en compte là aussi, on n'arrête pas de nous dire les filles, la moyenne des filles ».

Dans cet extrait le dialogue s'instaure autour de l'indifférenciation des critères et indicateurs pour évaluer les productions des filles au regard de celles des garçons dans des épreuves d'évaluation se déroulant le plus souvent en équipes mixtes. Les deux enseignants s'entendent pour considérer qu'il existe une différence entre "une motricité de fille et une motricité de garçon » dont il faut tenir compte pour rendre l'évaluation la plus équitable possible en modulant la note sur l'efficacité individuelle: «tu n'hésites pas à monter ...1 ou 2 points sur la note». 

diverses. Il est ainsi nécessaire de suivre les prescriptions institutionnelles en respectant strictement le référentiel qui ne fait pas la distinction entre les niveaux de jeu des filles et des garçons sachant que "c'est pas simple à écrire ». Par ailleurs, il y a obligation de porter une attention particulière aux filles pour que ne soit pas observé de décrochage dans les moyennes au baccalauréat entre garçons et filles. Cette injonction sur les notes des filles est notamment dictée par le corps d'inspection, elle est fortement intériorisée par ces enseignants soucieux de la réussite des élèves : faut que ça soit pris en compte là aussi, on n'arrête pas de nous dire les filles, la moyenne des filles ». Notons que dans cette IS, le discours de l'instructeur n'est pas uniquement adressé à son sosie, un sur-destinataire est également présent (Clot, 2008). Cette présence se manifeste quand sont évoquées les consignes délivrées par la hiérarchie et plus spécifiquement par les inspecteurs pédagogiques (" on n'arrête pas de nous dire ») pour aligner les notes des filles sur celles des garçons.

35 Le deuxième type de prescriptions qui affecte les choix des deux évaluateurs relève $d u$ registre des prescriptions remontantes. Les deux enseignants s'appuient sur les mêmes constats empiriques : à niveau égal les filles "c'est un petit moins», «la puissance» des tirs et des passes n'est pas la même que celle des garçons ( « des fois on a tendance à l'oublier un petit peu»). Il s'avère donc indispensable de compenser cette forme de «déficit» en valorisant le niveau de jeu atteint par les filles, par exemple dans l'IS $n^{\circ} 4$ l'instructeur précise «qu'on ne base pas son évaluation des filles sur des critères techniques purs mais plus sur des intentions, sur la compréhension du jeu ». Ce point de vue est aussi partagé dans les IS n ${ }^{\circ} 1$ et 2 avec cependant des appréciations plus sévères tenues par les quatre enseignantes sur l'engagement des filles et leurs compétences techniques, l'expression "plantes vertes » est d'ailleurs utilisée dans ces deux IS pour qualifier les filles en difficulté dont le niveau de jeu se rapproche de celui des débutants. Sont ainsi incorporés sans que ces enseignants en aient forcément conscience, des modèles, représentations, comportements, valeurs, rôles, positions, stéréotypes liés aux rapports de sexe.

Quelles sont les raisons qui poussent les enseignants à caractériser la "motricité des filles " de cette manière et à procéder à des arrangements pour ne pas pénaliser les filles par rapport aux garçons? Les résultats permettent d'avancer l'hypothèse selon laquelle l'acte d'évaluation en EPS serait fondé sur des indices caractéristiques d'une sportivité masculine qui expliquerait, en partie, les différences de notes entre filles et garçons (Vigneron, 2006). Les enseignants développeraient ainsi des perceptions et des attentes différentes selon le sexe des élèves. Ces attentes fonctionnent sur le mode de la prophétie, elles influencent le jugement de l'évaluateur dans la mesure où il construit son évaluation en fonction de ce qu'il s'attend à voir apparaître chez tel (le) ou tel (le) élève. Le modèle de référence de l'évaluateur se déclinerait donc au masculin y compris chez les enseignantes femmes (Bergé, 2000) qui n'arriveraient pas à admettre le peu d'appétence qu'ont certaines adolescentes pour l'activité physique. Ces enseignantes ont en effet en commun d'avoir fréquenté le milieu sportif de manière assidue, elles y ont réussi voire excellé ce qui est loin d'être le cas de l'ensemble des élèves de lycée. De fait, en adoptant des critères proches des traits caractéristiques des «modes d'organisation en sports collectifs » chez les garçons (Vigneron, 2005, p. 141) leur jugement devient plus sévère à l'égard des filles. Pour autant, les normes adoptées par la profession contraignent les enseignants à être "plus cool et plus compréhensif» (IS $\left.n^{\circ} 4\right)$ pour ne pas que l'EPS donne 
l'image d'une discipline scolaire qui accentuerait les inégalités de réussite entre filles et garçons.

\section{Discussion et conclusion}

L'objectif de l'article visait à documenter l'activité évaluative d'enseignants d'EPS de lycée afin de rendre intelligible le curriculum tel qu'il s'évalue en convoquant les cadres et les outils de l'analyse du travail. Les résultats ont montré que bien que confrontés au cadre très contraignant des référentiels, les enseignants se dotent d'une certaine autonomie qui leur permet de prendre des distances par rapport aux prescriptions officielles. Ce résultat peut de prime abord surprendre car les prescriptions descendantes qu'elles émanent du législateur mais aussi des corps d'inspection pourraient laisser penser que les enseignants n'ont pas de véritables marges de manœuvre dans la mesure où les référentiels de certification ont pour fonction de calibrer voire d'harmoniser les actes d'évaluation afin que le principe d'équité soit respecté. Comme le souligne Amigues (2003), l'écart entre le travail prescrit et le travail réalisé est au cœur de l'activité professorale, il ne faut pas y voir un signe d'incompétence chez l'enseignant voire une incompréhension de sa part des injonctions institutionnelles qui sont censées orienter son activité mais plutôt comme une activité en tension où le sujet doit faire des choix et des compromis entre différentes sources de prescriptions.

L'étude de l'activité réelle des évaluateurs montre ainsi que les enseignants ont tendance à jouer avec les règles institutionnelles, le curriculum tel qu'il s'évalue est parfois relativement éloigné de ce qu'en attend le législateur. Les enseignants n'appliquent pas mécaniquement ce qu'on leur demande d'évaluer, ils font preuve d'adaptation en fonction des contraintes auxquelles ils ont à faire face et se livrent à des arrangements multiples sans remettre en cause fondamentalement le travail qui leur est prescrit. Si leur activité est empêchée au motif qu'ils éprouvent des difficultés à respecter à la lettre les textes officiels, ils procèdent à des arbitrages qui sont parfois source de controverses. Ces arbitrages les amènent à "tordre » les référentiels qui sont à leur disposition afin de surmonter les obstacles qu'ils ont à affronter (profils d'élèves à évaluer, niveaux d'exigence attendus, notation des filles et des garçons ...) tout en s'accordant sur un certain nombre de principes d'action communs organisant leur activité d'évaluation. On peut ainsi faire l'hypothèse que les gestes du métier qui sont à l'œuvre quand les enseignants d'EPS évaluent sont l'expression d'un genre professionnel (Clot \& Faïta, 2000). Ce genre professionnel qui s'incarne dans un collectif de travail se caractérise par une expérience professionnelle commune, une sorte de mémoire collective qui permet d'accumuler les gestes du métier reconnus de tous, auxquels on peut se référer et que l'on peut faire évoluer en fonction de ses propres façons de faire.

La technique de l'instruction a par ailleurs permis d'interroger le rapport singulier et personnel qu'entretiennent les enseignants vis-à-vis de leur activité évaluative (David \& Amarouche, 2007) et de pointer dans cette activité les détails du métier d'enseignant d'EPS quand celui-ci fait usage des référentiels de certification. Dans le cadre de cet article l'instruction au sosie a principalement été utilisée dans une visée de production de connaissances, elle peut cependant être mobilisée en formation car elle est l'occasion de mettre en débat au sein d'un collectif de travail les gestes ordinaires du métier relatifs à l'évaluation du curriculum en EPS. L'instruction au sosie permet ainsi de provoquer un dialogue entre professionnels et d'engendrer du développement. Elle pousse le sujet, en 
dialoguant avec un pair, à se poser des questions nouvelles sur son activité et à la revisiter.

\section{BIBLIOGRAPHIE}

Amigues, R. (2003). Pour une approche ergonomique de l'activité enseignante. Skholê, 1, 5-16.

Amigues, R., \& Lataillade, G. (2007). Le travail partagé des enseignants : rôle des prescriptions et dynamique de l'activité enseignante. Actualité de la Recherche en Education et en Formation, Strasbourg.

Bergé, F. (2000). Ecarts de notation entre garçons et filles dans la Loire. In B. David, Equité et arrangements évaluatifs. Certifier en Education Physique et Sportive. www.inrp.fr/publications.

Brau-Antony, S. (2000). Etude des référentiels d'évaluation : l'exemple du volley-ball. In B. David (Éd.), Education Physique et Sportive. La certification au baccalauréat (pp. 57-75). Documents et travaux de recherche en éducation, 39. Paris : INRP.

Brau-Antony, S. (2001). Les conceptions des enseignants d'éducation physique et sportive sur l'enseignement et l'évaluation des jeux sportifs collectifs : résultats d'une enquête. Revue STAPS, $56,93-108$.

Cardinet, J. (1987). Une évaluation adaptée aux démarches souples. Cahiers pédagogiques, 256, 36-38.

Carnus, M.-F., \& Terrisse, A. (2006). La compétence à évaluer : une compétence révélatrice de la professionnalité des enseignants d'EPS. Etude de cas et perspectives pour la formation. Savoirs, 12 , 56-74.

Cleuziou, J.-P. (1996). Mesure de l'asymétrie et cotation des performances. Science et motricité, 28, 44-52.

Clot, Y. (2008). Travail et pouvoir d'agir. Paris : PUF.

Clot, Y. (2013). Développer le métier : le collectif dans l'individu. In B. Carnel \& J. Moniotte (Éds), Intervention, Recherche et Formation : quels enjeux, quelles transformations? (pp. 24-36). Actes du $7^{\mathrm{e}}$ Colloque international ARIS.

Clot, Y., \& Faïta D. (2000). Genres et styles en analyse du travail. Concepts et méthodes. Travailler, $4,7-42$.

Daniellou, F. (2002). Le travail des prescriptions. $37^{\mathrm{e}}$ congrès Self 2 . Les évolutions de la prescription, Aix en Provence.

David, B. (2000). Education Physique et Sportive. La certification au baccalauréat. Documents et travaux de recherche en éducation, 39. Paris : INRP.

David, B., \& Amarouche, P. (2007). Approche clinique de l'activité évaluative de professeurs stagiaires d'éducation physique et sportive. eJRIEPS, 9, 4-17.

David, B., \& Brau-Antony, S. (2002). Les référentiels d'évaluation comme révélateurs des conceptions des enseignants. Avanté, 8, 69-84. 
De Ketele, J.-M. (2011). L'évaluation et le curriculum : les fondements conceptuels, les débats, les enjeux. Les Dossiers des Sciences de l'Education, 25, 89-106.

Dolz, J., Jacquin, M., \& Schneuwly, B. (2006). Le curriculum enseigné en classe de français en secondaire : une approche à travers des objets enseignés. In F. Audigier, M. Crahay \& J. Dolz (Éds), Curriculum, enseignement et pilotage (pp. 143-164). Bruxelles : De Boeck.

Figari, G. (1994). Evaluer : quel référentiel ? Bruxelles : De Boeck.

Grisay, A. (1989). Améliorer l'évaluation-bilan à l'école primaire. Mesure et évaluation en éducation, 11(4), 5-24.

Hubert, B. (2013). De l'usage des référentiels par les enseignants d'Education Physique et Sportive dans le cadre de l'évaluation certificative au baccalauréat. Mémoire de master 2 CIREF. Université Reims Champagne-Ardenne.

Lenoir, Y. (2006). Du curriculum formel au curriculum enseigné : comment des enseignants québécois du primaire comprennent et mettent en œuvre le nouveau curriculum de l'enseignement primaire. In F. Audigier, M. Crahay \& J. Dolz (Éds), Curriculum, enseignement et pilotage (pp. 119-141). Bruxelles : De Boeck.

Lenoir, Y. (2011). Conséquences des conceptions curriculaires actuelles sur les modes évaluatifs. Les Dossiers des Sciences de l'Education, 25, 13-28.

Leplat, J. (1997). Regards sur l'activité en situation de travail. Paris : PUF.

Ministère de l'Education Nationale, de l'Enseignement Supérieur et de la Recherche. Bulletin Officiel spécial $n^{\circ} 5$ du 19 juillet 2012. Evaluation de l'Education Physique et Sportive aux baccalauréats de l'enseignement général et technologique.

Oddone, I., Rey, A., \& Briante, G. (1981). Redécouvrir l'expérience ouvrière. Vers une autre psychologie du travail. Paris : Editions sociales.

Merle, P. (1996). L'évaluation des élèves. Enquête sur le jugement professoral. Paris : PUF.

Noizet, G., \& Caverni, J.-P. (1978). Psychologie de l'évaluation scolaire. Paris : PUF.

Perrenoud, P. (1984). La fabrication de l'excellence scolaire: du curriculum aux pratiques d'évaluation. Genève : Droz.

Saujat, F. (2002). Ergonomie de l'activité enseignante et développement de l'expérience professionnelle : une approche clinique du travail de professeur. Thèse de doctorat, non publiée. Université AixMarseille 1.

Sensevy, G. (2007). Des catégories pour décrire et comprendre l'action didactique. In G. Sensevy \& A. Mercier (Éds), Agir ensemble. L'action didactique conjointe du professeur et des élèves (pp. 13-49). Rennes : Presses Universitaires de Rennes.

Six, F. (1999). De la prescription à la préparation du travail : apports de l'ergonomie à la prévention et à l'organisation du travail sur les chantiers du bâtiment. Note de synthèse pour l'habilitation à diriger des recherches, Université Charles de Gaulle Lille 3.

Vigneron, C. (2005).Une improbable réussite des filles en EPS : l'exemple des sports collectifs. In G. Cogérino (Éd.), Filles et garçons en EPS (pp. 125-162). Paris : Edition Revue EPS.

Vigneron, C. (2006). Les inégalités de réussite en EPS entre filles et garçons : déterminants biologiques ou fabrication scolaire? Revue française de pédagogie, 154, 111-124.

Vygotski, L. S. (1978). Mind in society. The development of higher mental processes. Cambridge : Harvard University Press. 


\section{RÉSUMÉS}

L'article a pour objectif de montrer qu'il est possible d'étudier le curriculum en Education Physique et Sportive (EPS) en l'abordant du point de vue des pratiques d'évaluation. Analyser l'activité évaluative des enseignants de lycée doit ainsi permettre de rendre intelligible le curriculum tel qu'il s'évalue. A cette fin ont été convoqués les cadres et les outils de l'analyse du travail (ergonomie et clinique de l'activité) afin de rendre compte de l'activité réelle des enseignants quand ils font usage des référentiels de certification prescrits par les textes officiels. Les quatre instructions qui ont été menées ont permis de mettre en évidence que les enseignants se dotent d'une certaine autonomie par rapport aux prescriptions officielles. Les enseignants ont tendance à jouer avec les règles institutionnelles, c'est pourquoi le curriculum évalué est parfois relativement éloigné de ce qu'en attend le législateur. On assiste notamment à une modification des exigences attendues selon le profil des élèves et à l'évaluation de compétences ne figurant pas explicitement dans le curriculum prescrit.

The article aims to show that it is possible to study the curriculum in Physical Education and Sports from the point of view of evaluation practices. To analyze evaluative activity of high school teachers must therefore help to make intelligible the curriculum as it assesses. For this purpose it's been called the frames and the tools of analysis of work (ergonomics and clinical activity) in order to realize the real activity of teachers when they make use of certification standards prescribed by the official texts. The four instructions that were carried out have highlighted that teachers develop certain autonomy with regard to official regulations. Teachers tend to play with the institutional rules, which is why the evaluated curriculum is sometimes relatively far from the expectations of legislator. We insist especially on the change of expected requirements according to the profile of the students and on the evaluation of competencies not explicitly listed in the prescribed curriculum.

\section{INDEX}

Mots-clés : évaluation, curriculum, activité, prescription

Keywords : assessment, activity

\section{AUTEURS}

\section{STÉPHANE BRAU-ANTONY}

URCA/ESPE Académie de Reims, CEREP EA 4692

\section{BENOÎT HUBERT}

Collège Arthur Rimbaud 